\title{
Self-Stabilizing Encapsulation through Fast Interfacial Polymerization of Ethyl $\alpha$-Cyanoacrylate: from Emulsions to Microcapsule Dispersions
}

Xiaodong Lian, Shenglong Liao, Xiao-Qi Xu, Shoupeng Zhang, and Yapei Wang*

Key Laboratory of Advanced Light Conversion Materials and Biophotonics, Department of Chemistry, Renmin University of China, Beijing 100872, P. R. China

Tables of Contents

1. Data section

2. Supplementary note: Size analysis of droplets prepared by DIP technique

3. Movies 


\section{Data section}

a
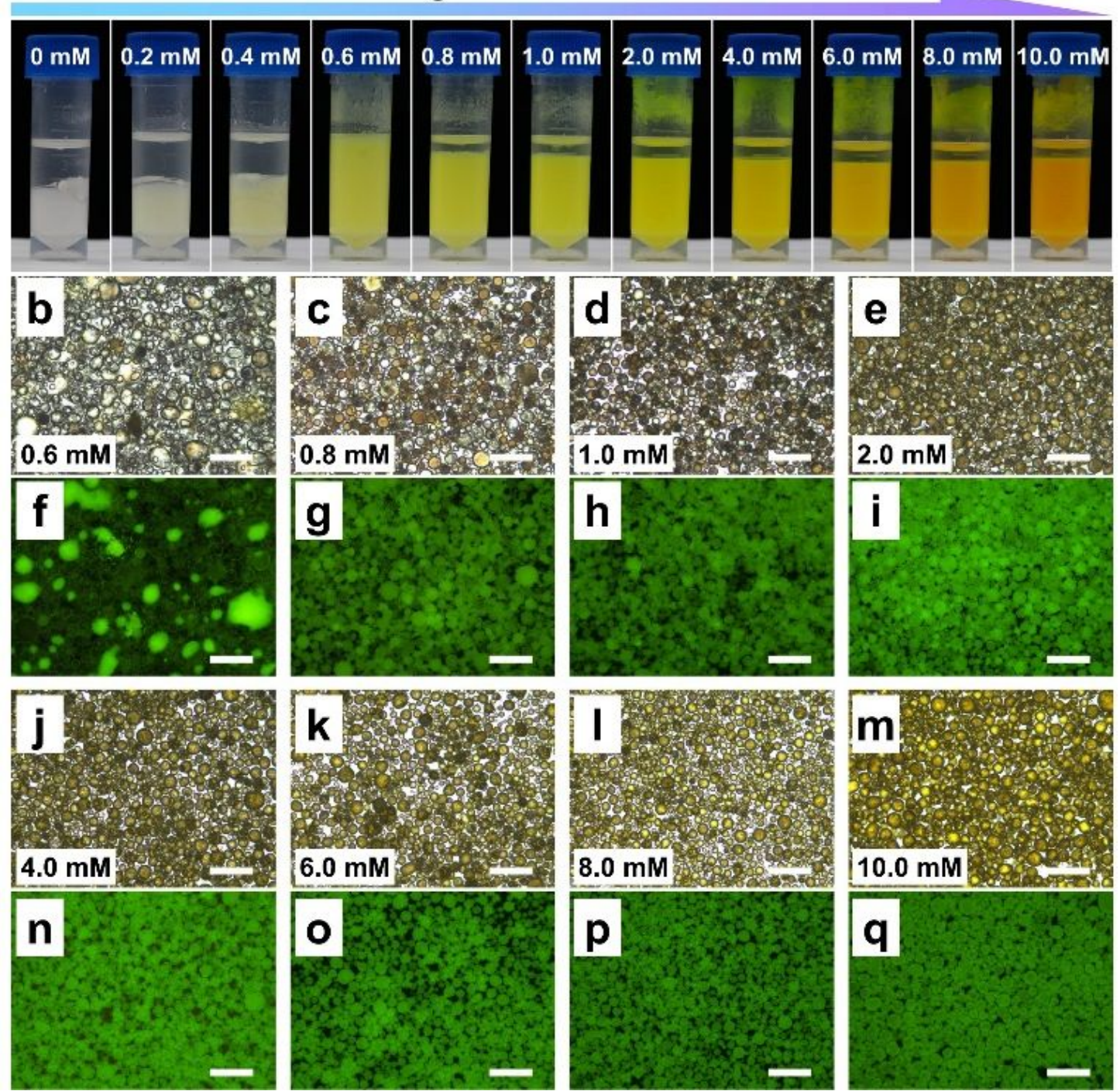

Figure S1. (a) Digital photographs of stable aqueous droplet dispersions (6 h after emulsification) with the water phase containing different concentrations of FS. (b-e, j-m) Bright-field and (f-i, n-q) fluorescent microscope images of stable aqueous droplet dispersions with the water phase containing different concentrations of FS. The oil phase is toluene loaded with $5.0 \mathrm{wt} \%$ ECA. Scar bar: $200 \mu \mathrm{m}$ for (b-q). 

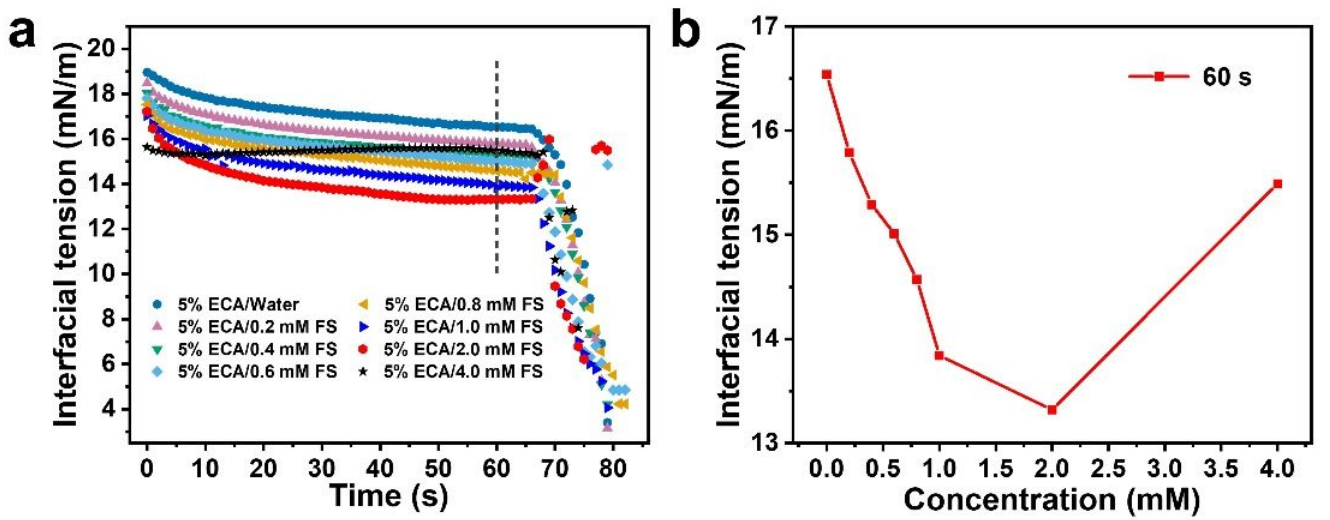

Figure S2. (a) Dynamic interfacial tension curves of the water-toluene interface with different concentrations of FS as the water phase and $5.0 \mathrm{wt} \% \mathrm{ECA}$ in toluene as the oil phase. (b) Corresponding dynamic interfacial tension values at 60th second as marked with a dashed line in (a).

a $0.4 \mathrm{mM}$ FS

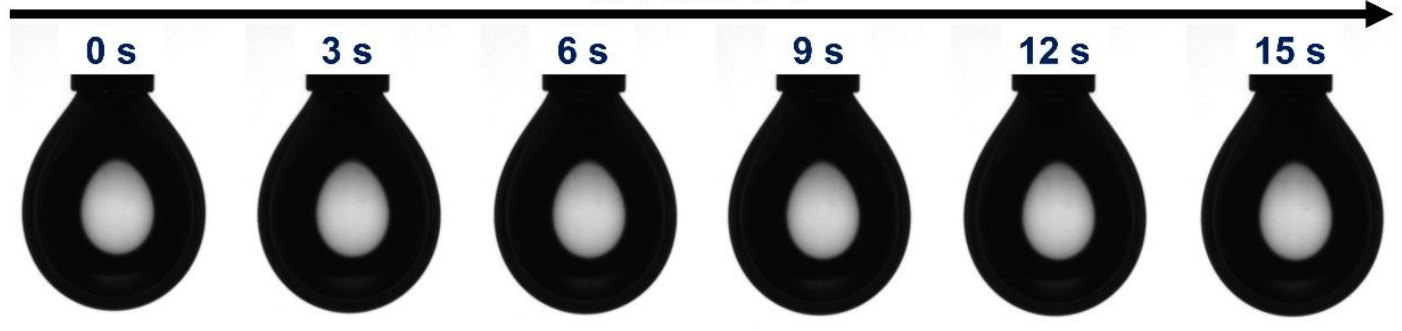

b

$4.0 \mathrm{mM}$ FS

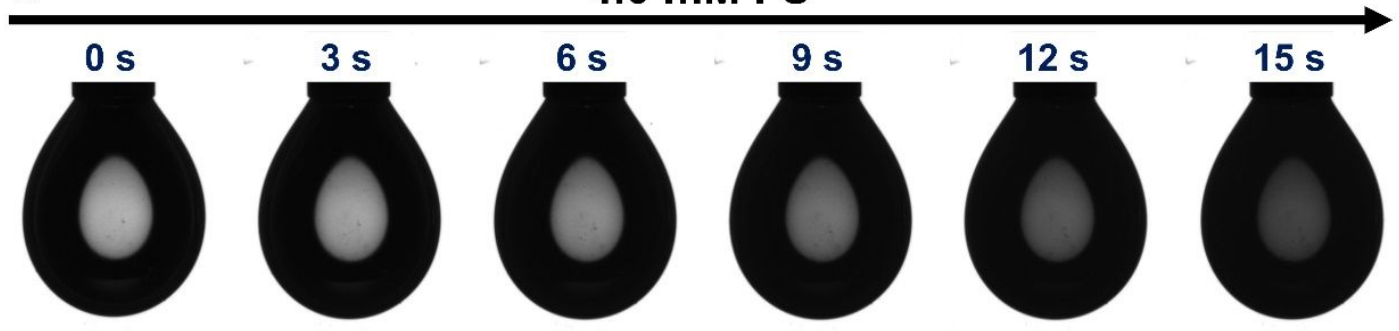

Figure S3. Optical images of pendant droplets of $0.4 \mathrm{mM} \mathrm{FS} \mathrm{(a)} \mathrm{and} 4.0 \mathrm{mM}$ FS (b) solution suspended in toluene loaded with $5.0 \mathrm{wt} \%$ ECA over time. 

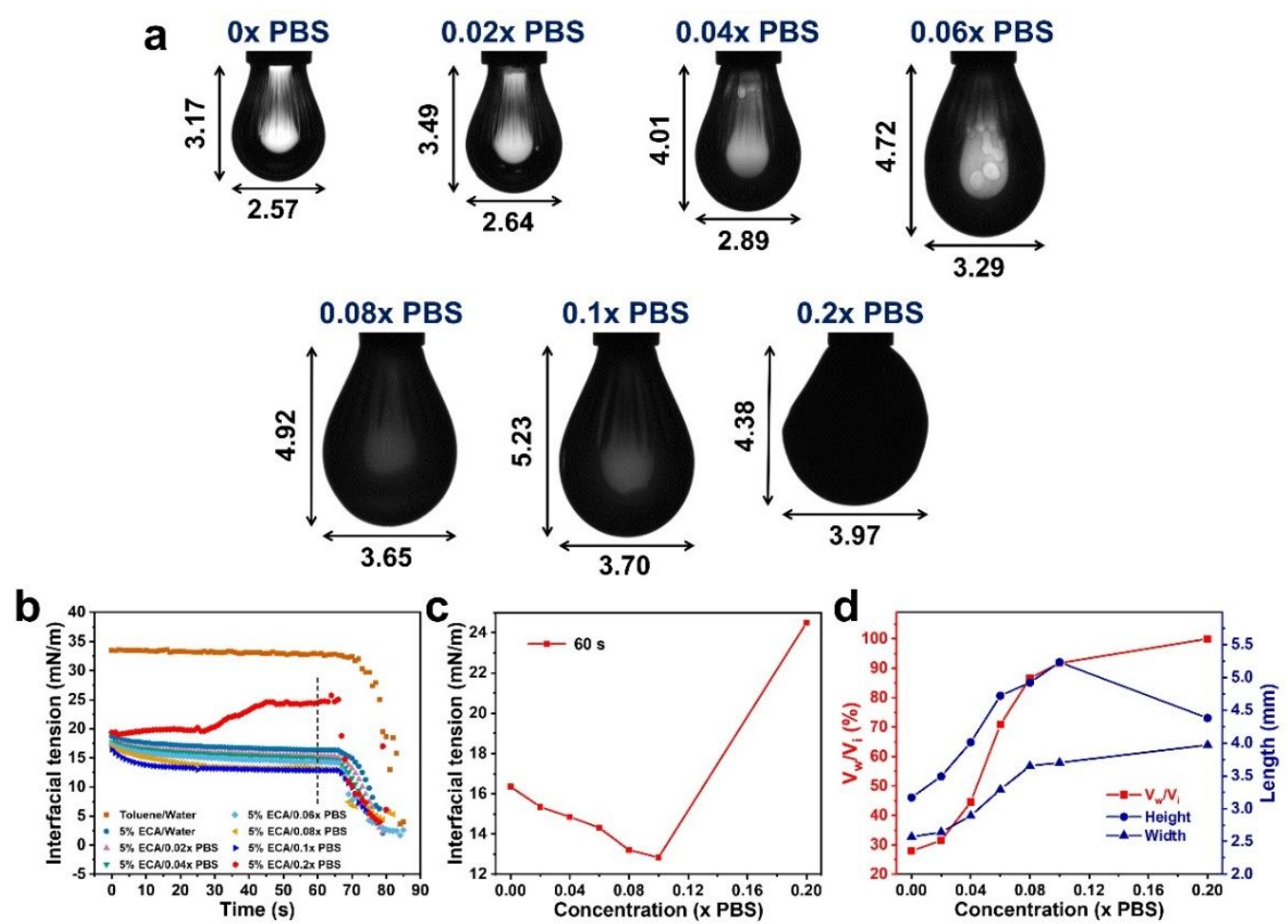

Figure S4. (a) Optical images of pendant droplets of PBS solution suspended in toluene (5.0 wt $\%$ ECA). (b) Dynamic interfacial tension curves of the water-oil interface. (c) Corresponding dynamic interfacial tension values at 60th second as marked with a dashed line in (b). (d) Compression ratios (red line) and droplet height/width analysis (blue lines) of the water-oil interface.
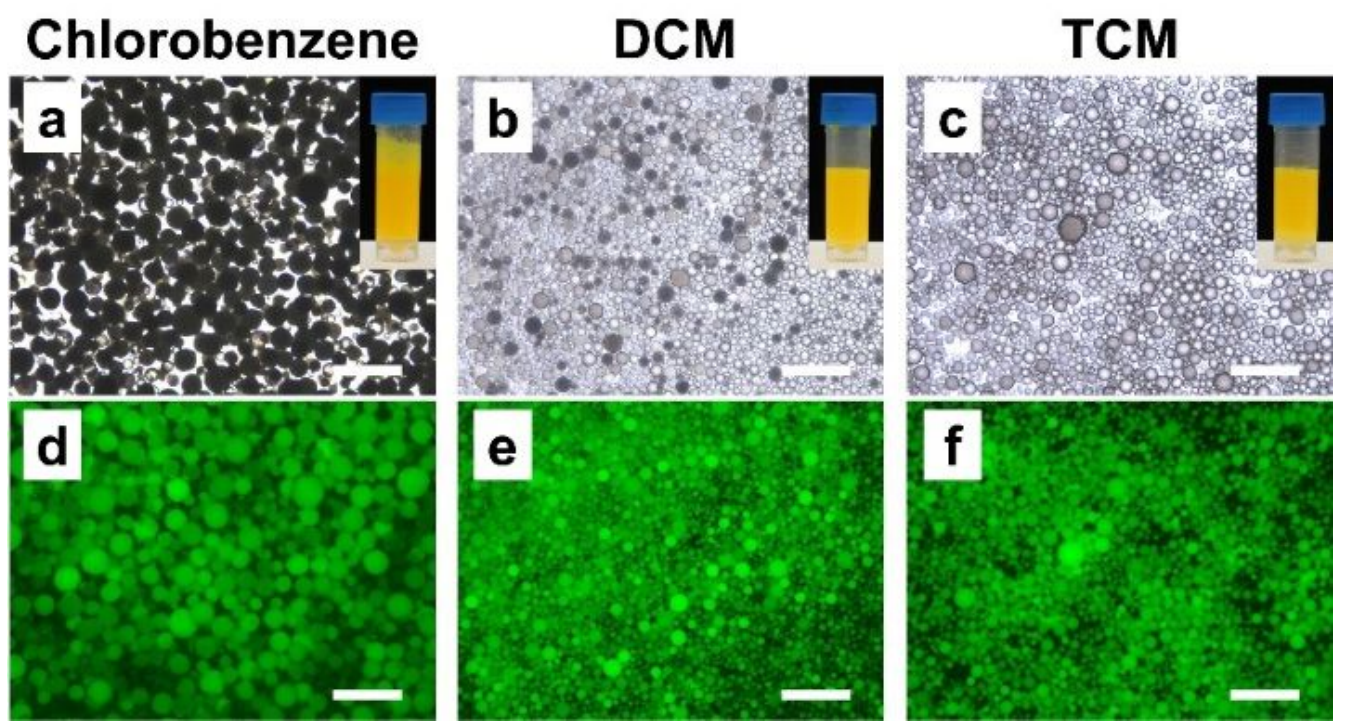

Figure S5. (a-c) Bright-field and (d-f) fluorescent microscope images of dispersed droplets with the water phase containing $4.0 \mathrm{mM}$ FS (scale bar: $200 \mu \mathrm{m}$ ) and the oil phase is $5.0 \mathrm{wt} \%$ ECA in $(\mathrm{a}, \mathrm{d})$ chlorobenzene, (b,e) DCM, and (c,f) TCM, respectively. Insets in (a-c) are digital photographs of the corresponding stable aqueous droplet dispersions in vessels. Scar bar: $200 \mu \mathrm{m}$. 


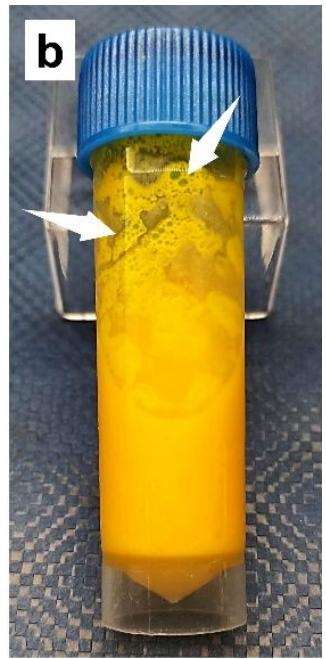

Figure S6. (a) Flow-process diagram of the re-homogenization process. (b) Digital photographs of the emulsion stabilized by the powder from the lyophilized droplet dispersion after the rehomogenization process. The concentration of FS in the water phase is $8.0 \mathrm{mM}$.

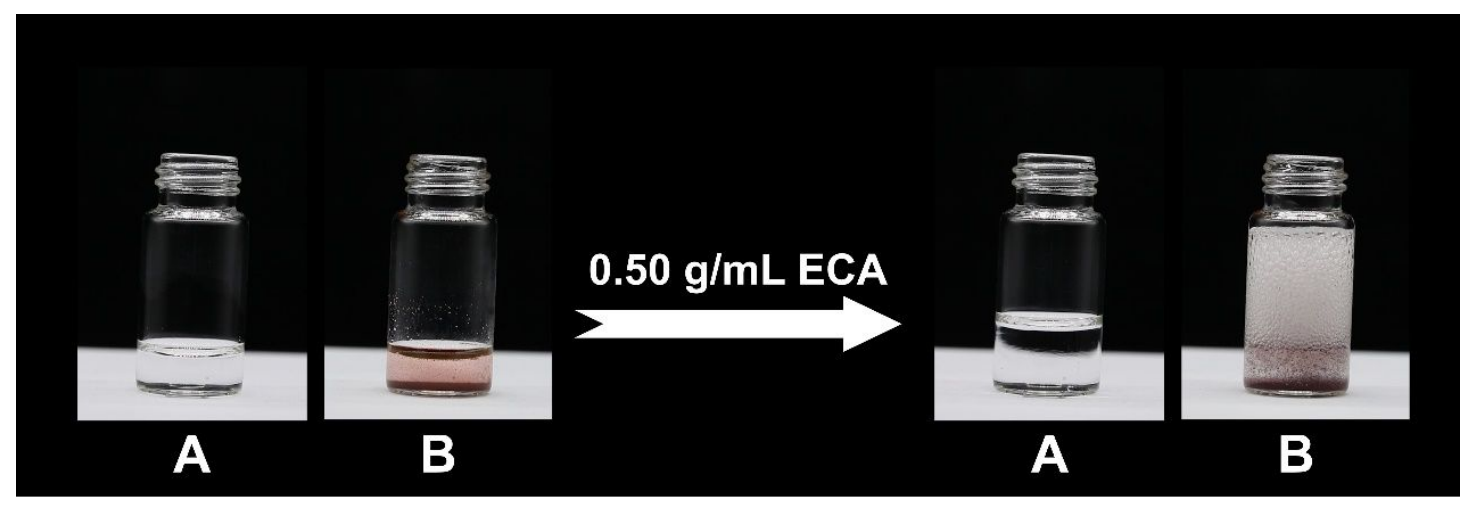

Figure S7. Digital photographs of solution polymerization by adding $1.0 \mathrm{~mL}$ of acetone loaded with $0.50 \mathrm{~g} / \mathrm{mL}$ ECA into $1.0 \mathrm{~mL}$ of acetone containing $25.0 \mathrm{mg}$ water (A) and $25.0 \mathrm{mg}$ FS (B), respectively.

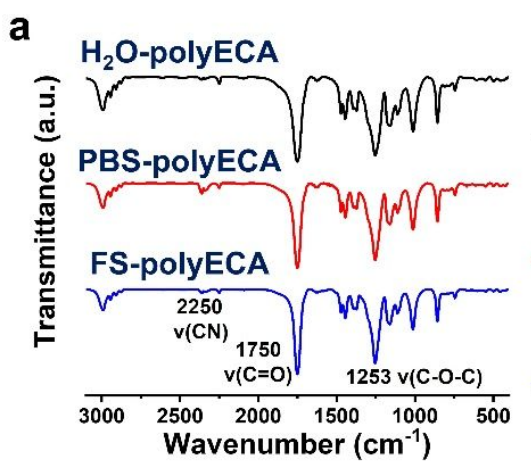

b

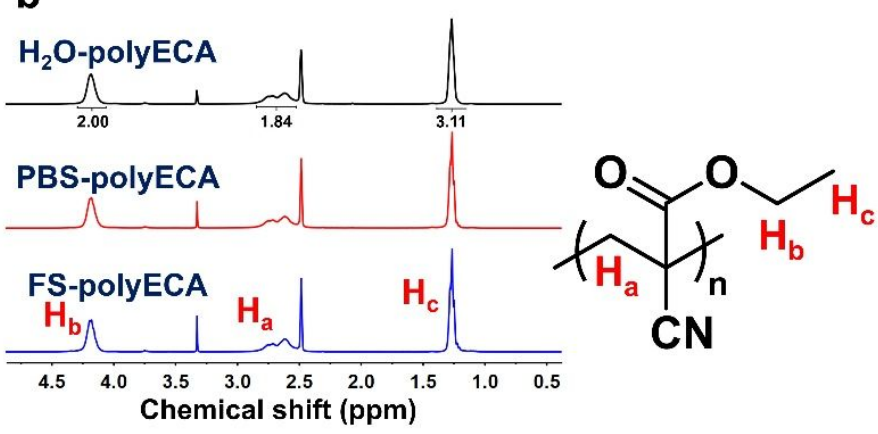

Figure S8. (a) FT-IR spectra, and (b) ${ }^{1} \mathrm{H}-\mathrm{NMR}$ spectra of polyECA initiated by pure water, PBS 
salt, and FS, respectively. The absorption peak of $\mathrm{C} \equiv \mathrm{N}$ stretching in polymer molecular environments located at $2250 \mathrm{~cm}^{-1}$; The absorption peak of saturated $\mathrm{C}=\mathrm{O}$ stretching located at $1750 \mathrm{~cm}^{-1}$; the absorption peak of single saturated $\mathrm{C}-\mathrm{O}-\mathrm{C}$ stretching locates at $1253 \mathrm{~cm}^{-1}$. The peaks at $\delta 4.2$ and $\delta 1.3 \mathrm{ppm}$ are due to protons of ethyl side group $-\mathrm{CH}_{3}$ and $-\mathrm{CH}_{2}-$, respectively, and the broad peaks located at $\delta 2.6-2.9 \mathrm{ppm}$ are from the backbone $-\mathrm{CH}_{2}-$.
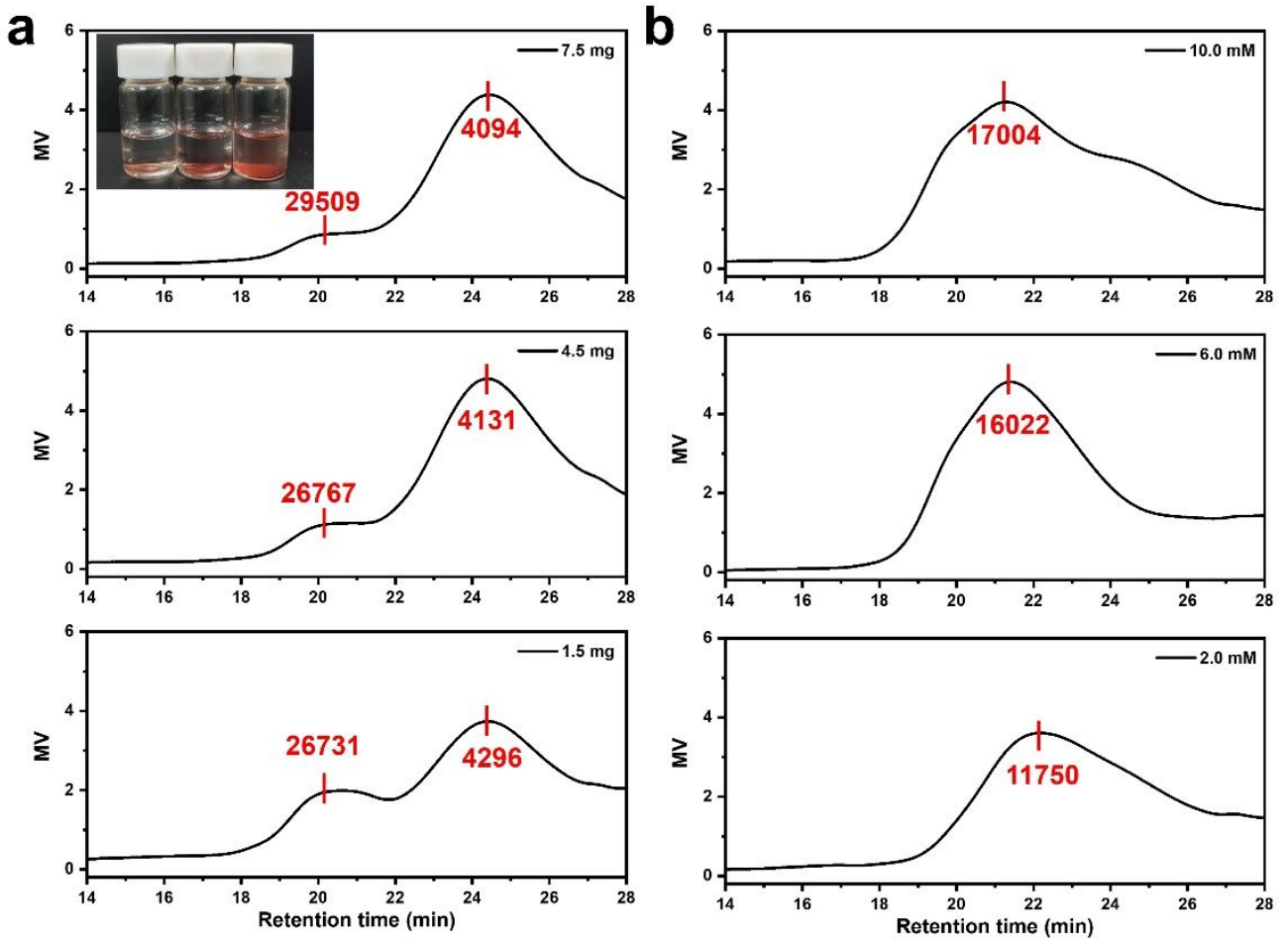

Figure S9. GPC curves of FS-polyECA products prepared by solution polymerization (a) and fast interfacial polymerization (b) at different initiator contents. Notably, in solution polymerization and fast interfacial polymerization, equimolar initiator was used as a comparative experiment. However, the solubility of FS in acetone is limited and $1.5 \mathrm{mg}$ is excessive. Inset in (a) is optical image after the solution polymerization, in which solid FS is observed for all samples. Left: $1.5 \mathrm{mg}$; Middle: $4.5 \mathrm{mg}$; Right: $7.5 \mathrm{mg}$. 


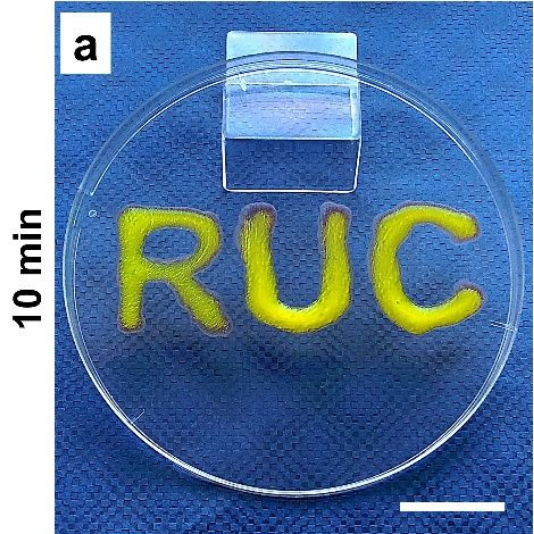

5.0 wt\% Abil EM90

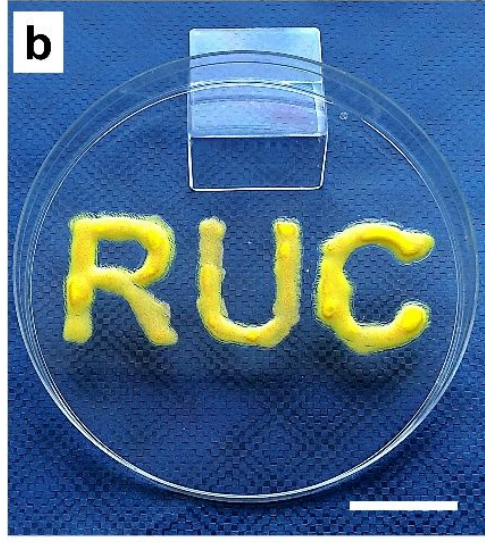

$5.0 \mathrm{wt} \% \mathrm{ECA}$

Figure S10. Digital photographs of the (a) traditional emulsion and (b) stable droplet dispersions prepared by FIP method on the surface of PS petri dishes after $10 \mathrm{~min}$. Scar bar: $2.0 \mathrm{~cm}$.
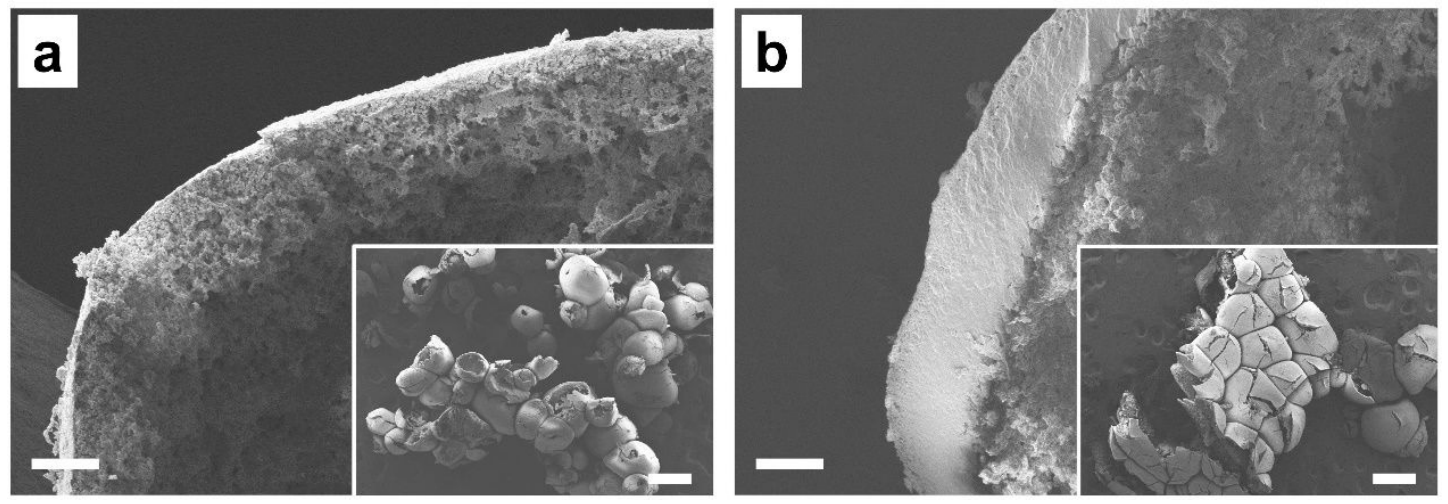

Figure S11. SEM images of freeze-dried HIPE-like systems with the water phase of (a) $4.0 \mathrm{mM}$ and (b) $8.0 \mathrm{mM}$ FS. Insets in (a) and (b) are images of a larger field of view. Scar bar: $20 \mu \mathrm{m}$ for (a, b) and $500 \mu \mathrm{m}$ for insets in (a, b). 

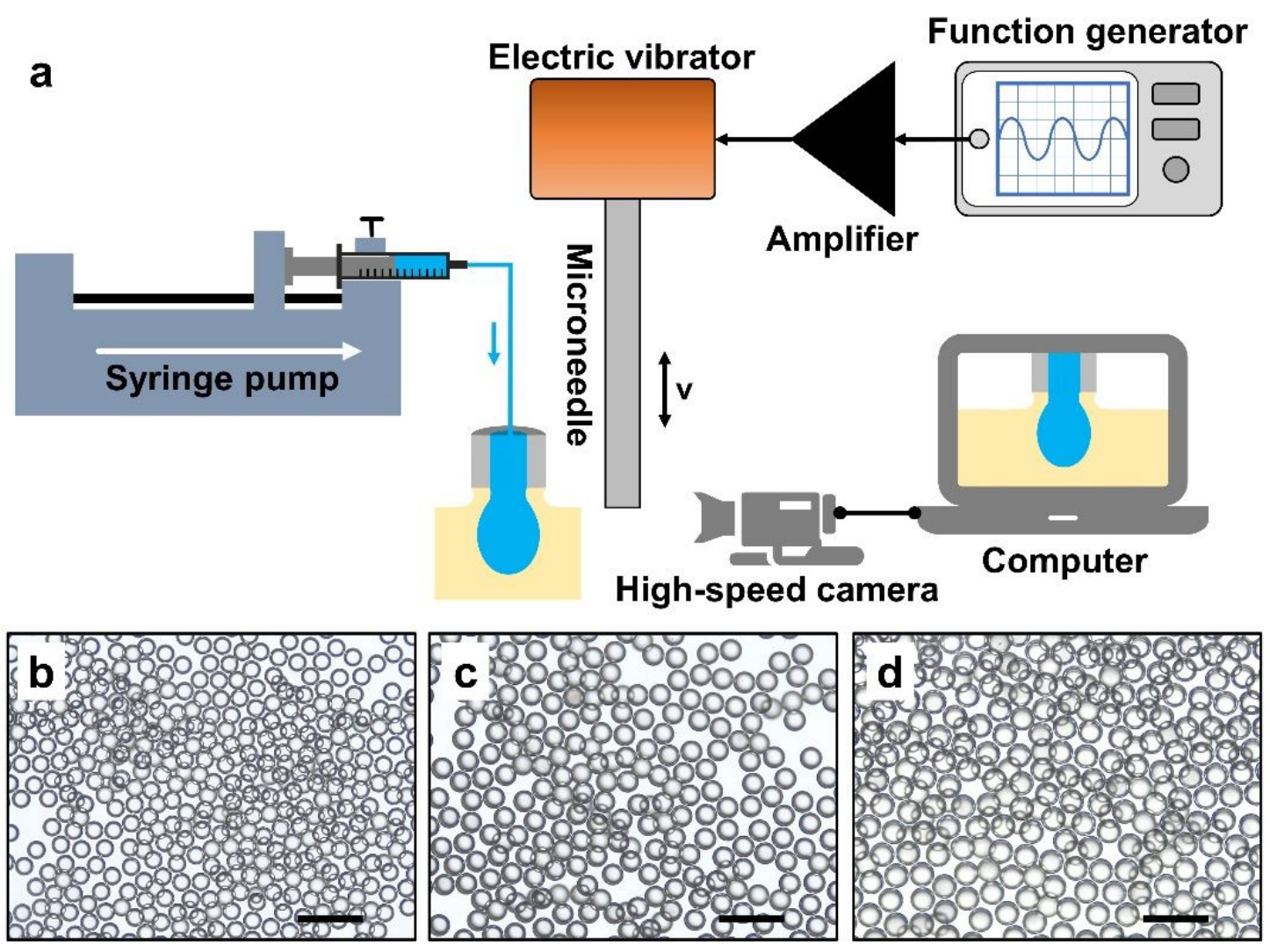

Figure S12. (a) Dynamic interfacial printing (DIP) system for the generation of monodispersed droplets. (b-d) Bright-field microscope images of uniform 4.0 mM FS droplets in toluene loaded with $5.0 \mathrm{wt} \%$ ECA under a large-field view, generated by a DIP technique at a flow rate of (b) 3.0, (c) 5.0 , and (d) $7.0 \mu \mathrm{L} \mathrm{min}{ }^{-1}$. Scar bar: $400 \mu \mathrm{m}$ for (b-d).

\section{Supplementary note: Size analysis of droplets prepared by DIP technique}

The theoretical size of monodispersed droplets produced by a DIP technique can be readily tailored by varying the flow rate $(\mathbf{Q})$ of the water phase or the vibration frequency $(\boldsymbol{f})$. Thus, we can calculate the volume of each monodisperse droplet $(\boldsymbol{V})$ according to Supplementary Equation (1).

$\boldsymbol{V}=\frac{\boldsymbol{Q}}{\boldsymbol{f}} \#(1$.

Water droplets in the oil phase tend to form a spherical shape due to the thermodynamic interfacial energy reduction. Based on the equation for calculating the volume of a sphere, the diameter of monodispersed droplets (D) produced by a DIP technique could be obtained as shown in Supplementary Equation (2).

$$
\boldsymbol{V}=\frac{\mathbf{4}}{\mathbf{3}} \boldsymbol{\pi}\left(\frac{\boldsymbol{D}}{\mathbf{2}}\right)^{\mathbf{3}} \#(2)
$$


Now considering the above equations, the diameter of monodispersed droplets (D) determined by the flow rate $(\mathbf{Q})$ and the vibration frequency $(\boldsymbol{f})$ can be concluded into Supplementary Equation (3).

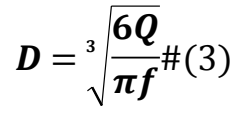

Herein, the vibration frequency $(f)$ was set as $60 \mathrm{~Hz}$, and only the flow rate is treated as an independent variable to compare the experimental value of droplet size with the ideal value. If the FIP method could not stabilized the generated monodispersed droplets, the experimental size distribution would be not consistent with the ideal condition. As expected, the experimental data are well consistent with the ideal condition, suggesting that the FIP method can rapidly and steadily stabilize the generated monodispersed droplets as given in Figure S12.

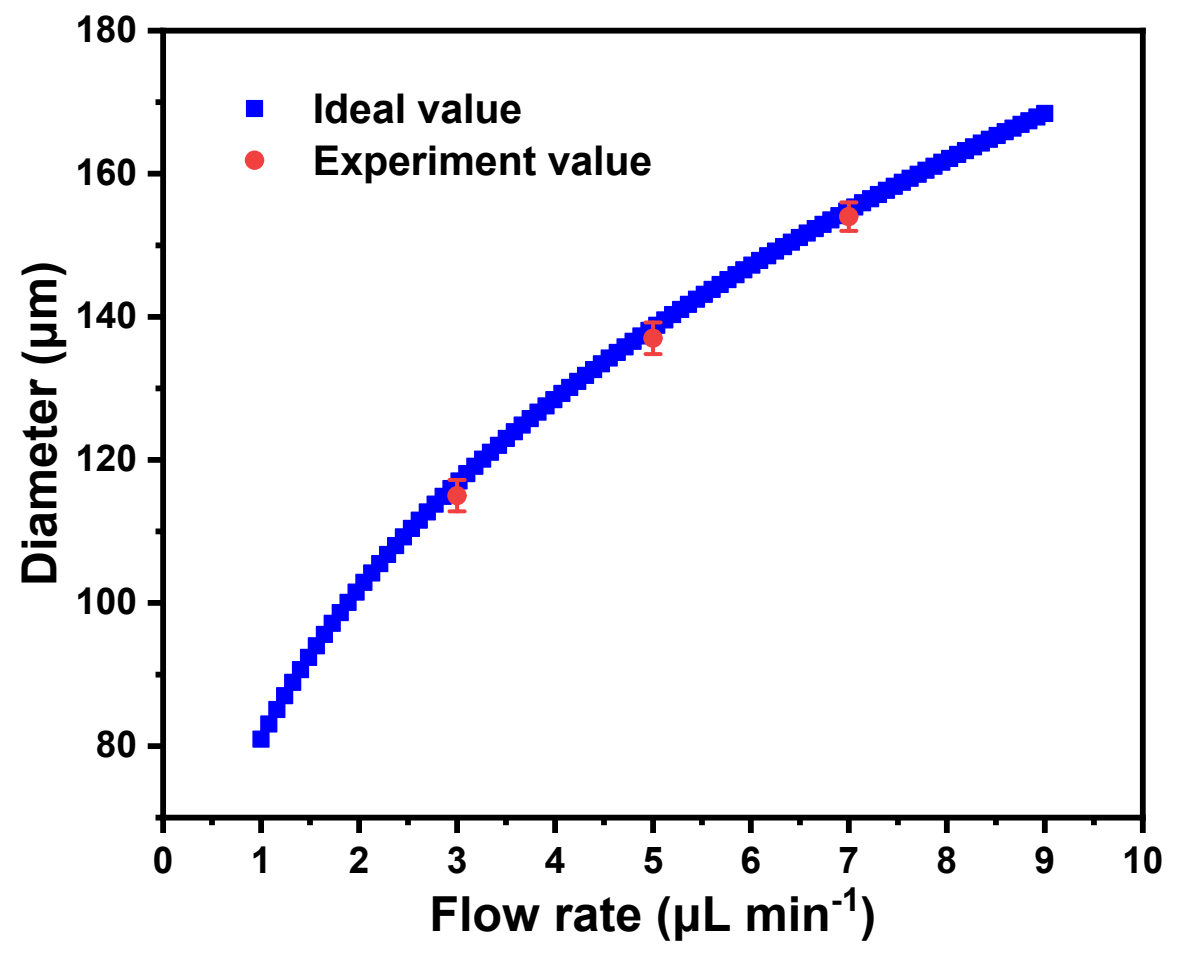

Figure S13. The plot of the ideal droplet size as a function of the flow rate.

\section{Movies}

Movie S1: Stability of millimetre-sized droplets of $4.0 \mathrm{mM} \mathrm{FS}$ solution in neat toluene and toluene containing $5.0 \mathrm{wt} \%$ ECA. The volume ratio of water to oil is 1:2. 
Movie S2: Receding process of droplets of FS solution with various concentrations via a pendant drop method. The initial volume is $40.0 \mu \mathrm{L}$, and the receding rate is $150 \mu \mathrm{L} \mathrm{min}^{-1}$.

Movie S3: Receding process of a droplet of $0.8 \mathrm{mM}$ FS solution via a pendant drop method. The initial volume is $40.0 \mu \mathrm{L}$, and the extraction rate is $150 \mu \mathrm{L} \mathrm{min}^{-1}$. About 10 seconds after the receding process, the reinjection experiment was conducted to show the viscoelastic polymer films at the interface.

Movie S4: Receding process of droplets of PBS solution with various concentrations via a pendant drop method. The initial volume is $40.0 \mu \mathrm{L}$, and the receding rate is $150 \mu \mathrm{L} \mathrm{min}^{-1}$.

Movie S5: Solution anion polymerization of ECA monomer initiated by $25.0 \mathrm{mg}$ water and 25.0 mg FS, respectively. Acetone was served as the good solvent. 